\title{
Avaliação da Função e Qualidade de Vida após Artroplastia Total da Anca por Diferentes Vias de Abordagem
}

\author{
Evaluation of the Function and Quality of Life after Total \\ Hip Arthroplasty by Different Approaches
}

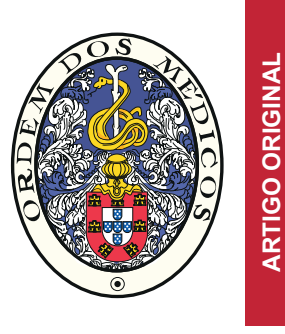

\author{
Paulo ARAÚJO $\triangle{ }^{1,2,3}$, Luís MACHADO², Duarte CADAVEZ², Lisete MÓNICO³, Filipa JANUÁRIO', Lisete LUÍS1, \\ Mafalda BÁRTOLO ${ }^{1}$ \\ Acta Med Port 2017 Sep;30(9):623-627 - https://doi.org/10.20344/amp.7834
}

\section{RESUMO}

Introdução: Avaliar a função e qualidade de vida em doentes submetidos a artroplastia total da anca fazendo distinção quanto às duas vias de abordagem (posterior/ântero-lateral) usadas pelo Serviço de Ortopedia do Centro Hospitalar de Leiria.

Material e Métodos: Estudo retrospetivo de 94 doentes sujeitos a artroplastia unilateral da anca, através do questionário Hip Osteoarthritis Outcome Score (HOOS LK 2.0), teste de Trendelenburg e avaliação da força muscular dos abdutores da anca com dinamómetro. Avaliaram-se os doentes aos seis meses, 12 meses, 18 meses e 24 meses pós-operatório.

Resultados: O estudo revelou que $97,9 \%$ doentes cumpriram programa de reabilitação. A evolução pós-cirúrgica (seis a 24 meses) mostrou ter resultados diferentes nas duas vias de abordagem. Aos seis meses os doentes operados pela via ântero-lateral apresentaram piores resultados quando comparados com a via posterior, nomeadamente Hip Osteoarthritis Outcome Score dor, Hip Osteoarthritis Outcome Score sintomas e Hip Osteoarthritis Outcome Score atividades da vida. Aos 24 meses, não parecem existir diferenças entre as duas vias de abordagem. Dos 94 doentes avaliados no estudo, o teste de Trendelenburg foi positivo em $31 \%$ dos doentes, sendo que $81,9 \%$ corresponderam a doentes operados pela via ântero-lateral. A força muscular dos abdutores da anca operada foi inferior na via ântero-lateral aos seis meses, 12 meses e aos 24 meses.

Discussão: Este trabalho evidenciou que nos primeiros seis meses pós-artroplastia total da anca, os doentes operados por via posterior apresentaram-se, de acordo com o questionário HOOS, menos sintomáticos, com melhor qualidade de vida e com menor impacto nas atividades de vida diária e no desporto e lazer quando comparados com os doentes operados por via ântero-lateral. Contudo, estas diferenças foram-se igualando ao longo dos 24 meses. Verificou-se ainda que os índices de força muscular dos músculos abdutores da anca foram claramente superiores nos doentes operados por via posterior aos seis meses, aos 12 meses e aos 24 meses comparativamente aos doentes operados por via ântero-lateral.

Conclusão: Aos 24 meses pós-artroplastia total de anca não parecem existir diferenças entre as duas vias de abordagem no que diz respeito à função e qualidade de vida. Contudo, verificou-se que os doentes operados pela via ântero-lateral apresentaram maiores défices de força muscular e maior percentagem de testes de Trendelenburg positivos.

Palavras-chave: Amplitude de Movimento Articular; Artroplastia Total da Anca; Qualidade de Vida; Recuperação de Função; Resultado do Tratamento

\section{ABSTRACT}

Introduction: To assess the function and quality of life in patients undergoing total hip arthroplasty distinguishing two surgical approaches (posterior / anterolateral) used by the Orthopedics department of Centro Hospitalar de Leiria.

Material and Methods: Retrospective study of 94 patients subject to unilateral hip replacement surgery, using the 'Hip Osteoarthritis Outcome Score' (HOOS LK 2.0) questionnaire, the Trendelenburg test and evaluation of muscle strength of the hip abductor muscles with dynamometer. Patients were evaluated at six months, 12 months, 18 months and 24 months after surgery.

Results: The study revealed that $97.9 \%$ patients completed the rehabilitation program. The postoperative evolution (six to 24 months) does not appear to have any differences in results when comparing both approaches. At six months the patient operated by the anterolateral approach showed worse results when compared with the posterior approach, in particular in Hip Osteoarthritis Outcome Score pain, in Hip Osteoarthritis Outcome Score symptoms and Hip Osteoarthritis Outcome Score activities of daily living. After 24 months, no differences between the two surgical approaches were found. Of the 94 patients evaluated, the Trendelenburg test was positive in $31 \%$ of patients of which $81.9 \%$ corresponds to patients operated by the anterolateral approach. Muscle strength of the abductors of the operated hip was clearly lower in the anterolateral approach at six months, 12 months, and 24 months.

Discussion: This study showed that, in the first six months after total hip arthroplasty, the patients operated by the posterior approach were, according to the HOOS questionnaire, less symptomatic, and presented with better quality of life and less impact on activities of daily living and in sport and leisure when compared with the patients operated by the anterolateral approach. However, these differences were matched over the 24 months. Moreover, the results of muscle strength of the abductor muscles of the hip were clearly superior in patients operated by the posterior approach at six months, at 12 months and at 24 months compared to the patients operated by the anterolateral approach.

Conclusion: After 24 months post total hip arthroplasty there are no differences between the two approaches with regard to function or quality of life. However, the patients operated by the anterolateral approach had greater muscle strength deficits and higher percentage of positive Trendelenburg test.

Keywords: Arthroplasty, Replacement, Hip; Quality of Life; Range of Motion, Articular; Recovery of Function; Treatment Outcome

\footnotetext{
1. Serviço de Medicina Física e de Reabilitação. Centro Hospitalar de Leiria. Leiria. Portugal.

2. Serviço de Ortopedia. Centro Hospitalar de Leiria. Leiria. Portugal.

3. Faculdade de Psicologia e de Ciências da Educação. Universidade de Coimbra. Coimbra. Portugal.

$\bowtie$ Autor correspondente: Paulo Araújo. paulocarauj089@gmail.com

Recebido: 08 de maio de 2016 - Aceite: 08 de maio de 2017 | Copyright $\odot$ Ordem dos Médicos 2017
} 


\section{INTRODUÇÃO}

A artroplastia total da anca é uma das técnicas de substituição de uma articulação do corpo humano com maior sucesso, a médio e longo prazo.

Nos últimos anos têm surgido novas vias de abordagem cirúrgica, cada vez menos invasivas, com a finalidade de diminuir o tempo de internamento e proporcionar ao doente uma rápida recuperação funcional. ${ }^{2}$ Há um consenso geral de que não interessa apenas o tamanho da incisão cirúrgica, mas também a preservação da integridade dos ventres musculares e das suas inserções para obter melhores resultados funcionais. ${ }^{3}$

Existem várias vias de abordagem descritas na literatura, sendo as mais usadas no Serviço de Ortopedia (SO) do Centro Hospitalar de Leiria ( $\mathrm{CHL}$ ) a via ântero-lateral e a via posterior. A via ântero-lateral apresenta menor taxa de luxação e melhor preservação anatómica dos músculos da anca. ${ }^{4} \mathrm{~A}$ principal desvantagem desta via é a diminuição da força muscular na abdução, devido à tenotomia do músculo glúteo médio, principal abdutor da anca. ${ }^{5,6}$ Apesar de rara, a lesão do nervo glúteo superior pode ocorrer durante o procedimento cirúrgico, podendo causar paralisia dos músculos glúteo médio, glúteo mínimo e do tensor da fáscia lata. ${ }^{6}$ Na via posterior a principal vantagem é a melhor exposição anatómica da articulação coxofemoral, sendo que esta via apresenta maiores taxas de luxação. ${ }^{7}$ Durante o procedimento cirúrgico pode ocorrer lesão do nervo ciático. ${ }^{8}$

Uma vez que o tipo de abordagem cirúrgica tem grande impacto na estabilidade e função muscular da anca é importante compreender que a força muscular diminui substancialmente nas primeiras semanas após a cirurgia, pelo que é necessário iniciar quanto antes um programa de reabilitação. ${ }^{9}$ Os principais objetivos do programa passam pelo ganho de força muscular, pela recuperação das amplitudes articulares, pela estabilização da articulação coxofemoral, pela recuperação da funcionalidade e uma mais rápida readaptação às atividades de vida diária. ${ }^{10}$

O principal objetivo deste estudo foi avaliar a existência de diferenças na função e qualidade de vida em doentes submetidos a artroplastia total da anca por duas vias de abordagem distintas (posterior e ântero-lateral).

\section{MATERIAL E MÉTODOS}

Foram incluídos neste estudo os doentes submetidos a prótese total unilateral da anca por doença degenerativa primária da anca. Os critérios utilizados para o diagnóstico de coxartrose foram a presença de dor na anca e a presença de alterações na radiografia da anca (diminuição da interlinha articular e/ou a presença de osteófitos). Todos os doentes acordaram em participar no estudo e deram o seu consentimento informado.

Foi feito um estudo retrospetivo. Foram avaliados diferentes doentes com tempos pós-cirúrgicos de seis meses, 12 meses, 18 meses e 24 meses

A escolha da técnica cirúrgica foi de acordo com a preferência do cirurgião, tendo em conta as vantagens e desvantagens das duas técnicas.

Foram usados como critérios de exclusão a presença de outras próteses ou implantes nos membros inferiores, sequelas de fraturas ósseas dos membros inferiores, displasia da anca ou doentes com patologia neurológica.

Os parâmetros avaliados neste estudo foram a força muscular dos músculos abdutores da anca operada e da anca contralateral, a aplicação do teste de Trendelenburg, o cumprimento do protocolo de reabilitação do Serviço de Medicina Física e de Reabilitação (SMFR) do CHL e foi usado o questionário Hip Osteoarthritis Outcome Score (HOOS LK 2.0), ${ }^{11}$ que avalia os doentes em cinco domínios (dor, sintomas, atividades de vida diária, desporto e lazer, e qualidade de vida). Este questionário apresenta os resultados num valor percentual de $0-100$, sendo que os valores

Tabela 1 - Programa de reabilitação do SMFR do CHL

\begin{tabular}{llll}
\hline Fase I & Fase II & Fase III & Fase IV \\
D1 - Alta internamento & S4 a S8 & S8 a S12 & S12 a S16 \\
\hline
\end{tabular}

- Controlo álgico:

- Analgésicos

- Anti-inflamatórios não esteróides

- Crioterapia estática

- Massagem manual

- Fortalecimento muscular isométrico dos flexores, extensores e abdutores da anca
- Estiramento do tensor da fáscia lata, tricípite sural, quadricípite, isquiotibiais, flexores da anca e adutores da anca

- Mobilização passiva da anca e ativaassistida do joelho, tíbio-társica e articulações do membro inferior não operado

- Fortalecimento dinâmico dos membros superiores

- Fortalecimento dinâmico do membro inferior não operado

- Treino de equilíbrio e marcha com - Treino de marcha com 1 canadiana a evoluir para marcha sem produtos de apoio andarilho ou canadianas

\begin{abstract}
Fortalecimento muscular dinâmico, sem carga, dos flexores, extensores e abdutores da anca
\end{abstract}

Fortalecimento muscular dinâmico, carga progressiva, dos flexores, extensores e abdutores da anca

Técnicas globais de ganho de amplitudes articulares (derivados Kabat) 
mais elevados significam melhores resultados funcionais.

O médico responsável pela avaliação destes parâmetros foi sempre o mesmo para evitar o risco de intervariabilidade.

A avaliação da força muscular dos músculos abdutores da anca operada e da anca contralateral foi mensurada com recurso a um dinamómetro de mola (escala máxima de $15 \mathrm{~kg}$ ). O doente foi colocado em decúbito lateral com a anca em posição neutra e o médico efetuou três medições seriadas da força de abdução de cada membro inferior sendo o resultado final a média das três medições.

O teste de Trendelenburg avalia a integridade funcional dos músculos abdutores da anca. O principal músculo testado é o glúteo médio. O teste é considerado positivo quando a bacia de um doente se inclina inferiormente, até aos 30 segundos, para o lado do membro inferior suspenso, significando que a fraqueza muscular está presente no membro inferior em apoio. ${ }^{11}$

O protocolo do SMFR do CHL consiste num programa de reabilitação com vista a obter controlo álgico, prevenir e vigiar as complicações pós-cirúrgicas, recuperar as amplitudes articulares e força muscular, assim como tornar o doente capaz de realizar marcha autonomamente, promovendo a sua reintegração e participação social. O protocolo encontra-se resumido na Tabela 1.

Realizamos a análise estatística com o SPSS-versão 22 , procedendo-se à análise descritiva e de inferência dos dados.

\section{RESULTADOS}

A amostra é composta por 94 doentes, sendo 48 operados pela via ântero-lateral (idade média de 69,7 \pm 9,1 anos) e 46 pela via posterior (idade média de 69,1 \pm 10,3 anos). O protocolo de reabilitação do SMFR do $\mathrm{CHL}$ foi cumprido em $97,9 \%$ dos doentes.

Em cada tempo pós-cirúrgico o número de doentes avaliados por cada via foi semelhante. Nas tabelas 2 e tabela 3 é apresentada a caracterização da amostra.
A comparação do questionário HOOS com as duas vias de abordagem mostrou diferentes resultados consoante o tempo pós-cirúrgico. A maior diferença foi verificada aos seis meses com os melhores resultados a serem obtidos através da via posterior, nomeadamente para o HOOS dor $(90 \pm 11,27)$, HOOS sintomas $(88,57 \pm 12,49)$ e HOOS atividades da vida diária $(82,43 \pm 17,8)$, quando comparados com o HOOS dor $(65,83 \pm 23,33)$, HOOS sintomas $(67,33$ $\pm 20,41)$ e HOOS atividades da vida diária $(57,83 \pm 20,82)$ dos doentes operados pela via ântero-lateral. Na tabela 4 são apresentados os resultados.

Aos 12 meses e 18 meses as diferenças entre as duas vias foram-se esbatendo nos vários domínios do questionário HOOS. Aos 12 meses apenas se destaca o HOOS para as atividades de vida diária na via posterior $(85,07 \pm 4,62)$ que apresenta melhores resultados que a via ântero-lateral $(66,53 \pm 25,66)$. Aos 18 meses verificou-se que o HOOS desporto e lazer na via posterior $(63,89 \pm 31,60)$ obteve meIhores resultados que na via ântero-lateral $(42,13 \pm 16,81)$.

Aos 24 meses pós-cirurgia os resultados do estudo mostraram uma aproximação entre as duas vias de abordagem, sendo os valores dos vários domínios do HOOS muito semelhantes nas duas vias de abordagem.

No que se refere à força muscular dos abdutores da anca, os melhores resultados foram obtidos pela via posterior, especialmente no que concerne aos seis meses, 12 meses e 24 meses (Tabela 4).

Dos 94 doentes avaliados, o teste de Trendelenburg foi positivo em cerca de $31 \%$, sendo que destes $81,9 \%$ foram operados pela via ântero-lateral (Tabela 5).

\section{DISCUSSÃO}

O objetivo deste estudo foi avaliar a função e qualidade de vida em doentes submetidos a artroplastia total da anca comparando as vias de abordagem posterior e ântero-lateral. Aos seis meses os doentes operados por via posterior apresentaram-se, de acordo com o questionário HOOS, menos sintomáticos, com melhor qualidade de vida e

Tabela 2 - Distribuição do número de doentes pela via de abordagem

\begin{tabular}{llccc}
\hline Tempo pós-cirurgia & Sexo & Via ântero-lateral & Via posterior & Total \\
\hline \multirow{2}{*}{$\mathbf{6}$ meses } & Masculino & 3 & 5 & 8 \\
& Feminino & 3 & 2 & 5 \\
$\mathbf{1 2}$ meses & Masculino & 9 & 5 & 14 \\
& Feminino & 6 & 9 & 15 \\
$\mathbf{1 8}$ meses & Masculino & 9 & 4 & 13 \\
\multirow{2}{*}{$\mathbf{2 4}$ meses } & Feminino & 3 & 8 & 11 \\
\multirow{2}{*}{ Total } & Masculino & 6 & 8 & 14 \\
\hline
\end{tabular}

Tabela 3 - Distribuição da média das idades (anos) pela via de abordagem

\begin{tabular}{lccccc}
\hline Via de abordagem & $\mathbf{n}$ & Mínimo & Máximo & Média & \multicolumn{1}{c}{ Desvio-padrão } \\
\hline Ântero-lateral & 48 & 46 & 86 & 69,71 & 9,08 \\
Posterior & 46 & 38 & 85 & 69,08 & 10,35 \\
\hline
\end{tabular}


Tabela 4 - Comparação do HOOS e força muscular $(\mathrm{kg})$ entre as duas vias de abordagem

\begin{tabular}{|c|c|c|c|}
\hline Tempo pós-cirurgia & Variáveis & Via ântero-lateral & Via posterior \\
\hline \multirow{6}{*}{6 meses } & HOOS dor & $65,83 \pm 23,33$ & $90 \pm 11,27$ \\
\hline & HOOS sintomas & $67,33 \pm 20,41$ & $88,57 \pm 12,49$ \\
\hline & HOOS AVD & $57,83 \pm 20,82$ & $82,43 \pm 17,8$ \\
\hline & HOOS desporto e lazer & $45,83 \pm 28,14$ & $66,07 \pm 28,38$ \\
\hline & HOOS qualidade vida & $54,33 \pm 33,89$ & $84,14 \pm 18,14$ \\
\hline & Média força muscular (kg) & $5,39 \pm 2,52$ & $9,67 \pm 3,18$ \\
\hline \multirow{6}{*}{12 meses } & HOOS dor & $69,96 \pm 27,97$ & $83,31 \pm 19,92$ \\
\hline & HOOS sintomas & $75,27 \pm 23,51$ & $86,07 \pm 14,57$ \\
\hline & HOOS AVD & $66,53 \pm 25,66$ & $85,07 \pm 14,62$ \\
\hline & HOOS desporto e lazer & $56,40 \pm 34,44$ & $75,47 \pm 19,59$ \\
\hline & HOOS qualidade vida & $59,80 \pm 22,44$ & $72,86 \pm 27,39$ \\
\hline & Média força muscular (kg) & $6,00 \pm 3,37$ & $8,93 \pm 2,68$ \\
\hline \multirow{6}{*}{18 meses } & HOOS dor & $70,40 \pm 9,14$ & $77,22 \pm 12,72$ \\
\hline & HOOS sintomas & $77,08 \pm 13,89$ & $80,42 \pm 18,52$ \\
\hline & HOOS AVD & $66,50 \pm 13,73$ & $74,58 \pm 18,73$ \\
\hline & HOOS desporto e lazer & $42,13 \pm 16,81$ & $63,89 \pm 31,60$ \\
\hline & HOOS qualidade vida & $48,65 \pm 24,20$ & $63,58 \pm 25,60$ \\
\hline & Média força muscular (kg) & $8,92 \pm 4,70$ & $7,81 \pm 4,28$ \\
\hline \multirow{6}{*}{24 meses } & HOOS dor & $81,28 \pm 18,41$ & $76,73 \pm 17,48$ \\
\hline & HOOS sintomas & $81,33 \pm 13,02$ & $79,62 \pm 16,26$ \\
\hline & HOOS AVD & $77,20 \pm 19,90$ & $73,08 \pm 20,27$ \\
\hline & HOOS desporto e lazer & $71,58 \pm 26,17$ & $54,81 \pm 26,41$ \\
\hline & HOOS qualidade vida & $64,07 \pm 23,82$ & $63,69 \pm 23,40$ \\
\hline & Média força muscular (kg) & $4,58 \pm 2,29$ & $7,40 \pm 2,96$ \\
\hline
\end{tabular}

Tabela 5 - Comparação entre a força muscular $(\mathrm{kg})$ e o teste de Trendelenburg

\begin{tabular}{lcc}
\hline Teste Trendelenburg & Força muscular abdutores da anca $\mathbf{( k g )}$ & Efetivos em \% \\
\hline Negativo & 8,28 & 69 \\
Positivo & 4,9 & $31^{*}$ \\
\hline
\end{tabular}

* 82,8 via ântero-lateral

menor impacto nas atividades de vida diária e no desporto e lazer. Aos 24 meses não parecem existir diferenças nos parâmetros de função ou de qualidade de vida. Contudo, os doentes operados pela via posterior apresentaram meIhores índices de força muscular dos músculos abdutores da anca aos seis meses, aos 12 meses e aos 24 meses. Já aos 18 meses, contrariamente ao expectável, os doentes operados por via ântero-lateral apresentaram melhores índices de força muscular. Estes resultados podem ser explicados pelo pequeno tamanho da amostra e por serem avaliados doentes distintos em cada tempo pós cirúrgico.

Palan et al compararam as abordagens cirúrgicas ântero-lateral e posterior na artroplastia total da anca no que diz respeito à função e qualidade de vida dos doentes através do questionário Oxford Hip Score (OHS), taxa de doentes com luxação da anca e a necessidade de cirurgia de revisão. Concluíram que, numa fase inicial, definida pelos autores entre os três meses e um ano pós-cirurgia, os doentes operados pela via posterior apresentam melhores resultados na dor e na funcionalidade do que os doentes operados pela via ântero-lateral. ${ }^{13}$ Verificaram também que, ao fim de dois anos e cinco anos, não havia diferenças nas duas vias de abordagem. Estes resultados são semelhantes aos encontrados neste estudo, que demonstrou haver doentes com maiores pontuações no HOOS aos seis meses do pós-operatório para a via posterior. Estas diferenças tornam-se menores ao fim de 24 meses.

Laffosse et al realizaram um estudo prospetivo e comparativo entre a via de abordagem ântero-lateral e posterior através da aplicação da escala visual analógica da dor, do questionário the Western Ontario and McMaster Universities Osteoarthritis Index (WOMAC) e do questionário OHS. Concluíram que, no pós-operatório, a dor é comparável nas duas vias de abordagem e que em termos de funcionalidade não havia diferenças às seis semanas, aos três meses e seis meses. ${ }^{14}$

Merril et al realizaram um estudo onde avaliaram o impacto da via de abordagem (ântero-lateral e posterior) relativamente à presença ou não de claudicação da marcha, luxação da prótese, tempo de internamento hospitalar e 
tempo de descarga pós-cirúrgica, ao final de 12 meses pós-cirurgia. Concluíram que não foram observadas diferenças estatisticamente significativas na claudicação da marcha entre as duas vias de abordagem, apesar de haver maior taxa de claudicação da marcha nos doentes operados pela via ântero-lateral. ${ }^{4}$

Quanto à avaliação da força muscular dos músculos abdutores da anca operada, verificou-se que a via posterior obteve melhores resultados que a via ântero-lateral em todos os tempos pós-cirúrgicos, com exceção aos 18 meses.

Segundo Onyemaechi et al na abordagem posterior não há atingimento do mecanismo abdutor da anca ao contrário da via ântero-lateral, na qual existe uma completa ou parcial desinserção do mecanismo abdutor, o que pode explicar as diferenças de força muscular nas duas vias de abordagem. ${ }^{6}$

No estudo de Hendrickx et al sobre o efeito da reabilitação funcional dos doentes submetido a artroplastia total da anca verificou-se que os doentes operados pela via ântero-lateral apresentaram maior claudicação da marcha nos três meses pós-operatório. ${ }^{5}$

As principais limitações deste estudo prenderam-se com a amostra reduzida de doentes. Outra limitação do estudo foi o facto de se tratar de um estudo retrospetivo em que os doentes avaliados em cada tempo pós-cirúrgico eram diferentes.

\section{REFERÊNCIAS}

1. Lemmey AB, Okoro T. The efficacy of exercise rehabilitation in restoring physical function following total hip replacement for osteoarthritis: a review. OA Musculoskeletal Med. 2013;1:13.

2. Gebel P, Oszwald M, Ishaque B, Ahmed G, Blessing R, Thorez F, et al. Process optimized minimally invasive total hip replacement. Orthop Rev. 2012;4:e3.

3. Heynem G, Donnelly W, Schieicher I, Turnbull A, Leong A. Preliminary results from an internacional, prospective, randomized, multicentre 1 year follow up total hip replacement (THR) study to evaluate a minimally invasive surgical technique. Orthopaedic Proc. 2004;86-B:482.

4. Ritter MA, Harty LD, Keating ME, Faris PM, Meding JB. A clinical comparison of the anterolateral and posterolateral approaches to the hip. Clin Orthop Relat Res. 2001;385:95-9.

5. Hendrickx C, Hertogh W, Daele U, Mertens P, Stassijns G. Effect of percutaneous assisted approach on functional rehabilitation for total hip replacement compared to anterolateral approach: study protocol for a randomized controlled trial. Trials. 2014;15:392.

6. Onyemaechi N, Anyanwu E, Obikili E, Ekezie J. Anatomical basis for surgical approaches to the hip. Ann Med Health Sci Res. 2014;4:487-94.

7. Edmunds CT, Boscainos PJ. Effect of surgical approach for total hip replacement on hip function using Harris Hip scores and Trendelenburg's test: a retrospective analysis. Surgeon. 2011;9:124-9.

8. Winther B, Husby S, Foss A, Wik S, Svenningsen S, Engdal M, et al. Muscular strength after total hip artroplasty, a prospective comparison of 3 surgical approaches. Acta Orthop. 2015;86:22-8.

\section{CONCLUSÃO}

Neste estudo, aos seis meses do pós-operatório os doentes submetidos a artroplastia da anca por via posterior apresentaram melhores resultados no HOOS, contudo parecem não existir diferenças aos 24 meses. A força do mecanismo muscular abdutor da anca foi superior em praticamente todos os tempos de avaliação na via posterior.

\section{PROTEÇÃO DE PESSOAS E ANIMAIS}

Os autores declaram que os procedimentos seguidos estavam de acordo com os regulamentos estabelecidos pelos responsáveis da Comissão de Investigação Clínica e Ética e de acordo com a Declaração de Helsínquia da Associação Médica Mundial.

\section{CONFIDENCIALIDADE DOS DADOS}

Os autores declaram ter seguido os protocolos do seu centro de trabalho acerca da publicação de dados.

\section{CONFLITOS DE INTERESSE}

Os autores declaram a inexistência de conflitos de interesses.

\section{FONTES DE FINANCIAMENTO}

Não existiram fontes externas de financiamento para a realização deste estudo.

9. Holm B, Thorborg K, Husted H, Kehlet H, Bandholm T. Surgery-induced changes and early recovery of hip-muscle strength, leg-press power. And functional performance after fast-track total hip arthroplasty: a prospective cohort study. Plos One. 2013;8:e62109.

10. Unlu E, Eksioglu E, Aydog E. The effect of exercice on hip muscle strenght, gait speed and cadence in patients with total hip arthroplasty: a randomized controlled study. Clin Rehabil. 2007;21:706-11.

11. Cavalheiro L, Gil JN, Nunes S, Ferreira PL, Gonçalves RS. Measuring health-related quality of life in patients with hip osteoarthritis and total hip replacement: adaption and validation of the hip disability and osteoarthritis outcome source LK 2.0 (HOOS 2.0) to the Portuguese culture. $18^{\text {th }}$ Annual Conference of the International Society of Quality of Life (ISOQOL 2011). Denver: 2011;40.

12. Downing D, Clark I, Hutchinson J, Colclough K, Howard P. Hip abductor strength following total hip arthroplasty-A prospective comparison of the posterior and lateral approach in 100 patients. Acta Orthop Scand. 2001;72:215-20.

13. Palan J, Murray W, Andrew J, Nolan J. Whitch approach for total hip arthrosplasty, anterolateral or posterior? Clin Orthop Relat Res. 2009;467:473-7.

14. Laffosse J, Chiron P, Molinier F, Benfasi H, Puget J. Prospective and comparative study of the anterolateral nini-invasive approach versus minimally invasive posterior approach for primary total hip replacement. Early results. Int Orthop. 2007;31:597-603. 